\title{
Estradiol Modulates the Pulsatile Secretion of Biologically Active Luteinizing Hormone in Man
}

\author{
Johannes D. Veldhuis* and Maria L. Dufau ${ }^{\ddagger}$ \\ *Division of Endocrinology and Metabolism, Department of Internal Medicine, University of Virginia School of Medicine, \\ Charlottesville, Virginia 22908; and \$Section of Molecular Endocrinology, Endocrine Reproduction Research Branch, National Institute \\ of Child Health and Human Development, National Institutes of Health, Bethesda, Maryland 20205
}

\begin{abstract}
We investigated the effects of estradiol on bioactive luteinizing hormone (LH) release in normal men using two complementary strategies: ( $i$ ) steady state intravenous infusions of estradiol at its endogenous production rate, and (ii) oral administration of the antiestrogen, tamoxifen $\mathrm{HCl}$. Immunoreactive and biologically active $\mathrm{LH}$ were monitored by radioimmunoassay and the rat interstitial cell testosterone bioassay, respectively. Estradiol infusions significantly suppressed mean plasma bioactive LH concentrations and decreased the bio/ immuno LH ratio. Conversely, antiestrogen treatment enhanced spontaneous bioactive LH pulse frequency, increased bioactive LH pulse amplitude, and augmented plasma intrapulse and interpulse bio/immuno LH ratios. Low-dose pulsed injections of exogenous gonadotropin-releasing hormone (GnRH) also increased plasma bio/immuno LH ratios. However, tamoxifen attenuated the ability of exogenous GnRH to further enhance the bio/immuno LH ratio, which suggests that endogenous LH release was already maximally enriched in LH bioactivity during antiestrogen administration.

We conclude that estradiol modulates the pulsatile secretion of LH molecules enriched in biological activity in man.
\end{abstract}

\section{Introduction}

Studies in experimental animals have suggested that sex steroid hormones may modulate the pituitary secretion of not only immunoactive gonadotropins but also biologically active hormones (1-4). In the rat, castration results in a disproportionate increase in the circulating concentrations of immunoreactive luteinizing hormone $(\mathrm{LH})^{1}$ as compared with biologically active LH (5). This relative decrease in the bio/immuno LH ratio is attenuated by testosterone and $5 \alpha$-dihydrotestosterone replacement. However, in the human, menopause and/or gonadal failure result in preferentially increased bioactive LH concentrations with high plasma bio/immuno LH

Address correspondence to Dr. Veldhuis, Box 202, Division of Endocrinology and Metabolism, Department of Internal Medicine, University of Virginia School of Medicine, Charlottesville, VA 22908.

Received for publication 27 October 1986 and in revised form 19 February 1987.

1. Abbreviations used in this paper: GnRH, gonadotropin-releasing hormone; LH, luteinizing hormone; RICT, rat interstitial cell testosterone.

\section{J. Clin. Invest.}

(C) The American Society for Clinical Investigation, Inc.

0021-9738/87/09/0631/08 $\$ 2.00$

Volume 80, September 1987, 631-638 ratios (6). The high level of bioactive $\mathrm{LH}$ in primary ovarian failure can be suppressed by oral administration of estrogen (7). The latter observation is consistent with the demonstration that young women of reproductive age who have high endogenous estradiol levels exhibit significantly lower plasma bio/immuno LH ratios than postmenopausal women or young men (8). The latter disparity between young women and men might reflect either a putative suppressive effect of estradiol on bioactive LH secretion in women, or a facilitative effect of testosterone on bioactive LH release in men. Accordingly, the exact nature of the effects of endogenous sex steroids on the bioactivity of circulating $\mathrm{LH}$ cannot be readily ascertained. Moreover, presumptive actions of sex steroids on physiological patterns of pulsatile bioactive $\mathrm{LH}$ release have not been defined.

In the present work, we have employed two complementary strategies to examine the role of estradiol in modulating the secretion of biologically active $\mathrm{LH}$ in the human. First, we investigated bioactive $\mathrm{LH}$ release in men subjected to steady state estradiol infusions. Secondly, we administered tamoxifen hydrochloride, a pharmacological antagonist of estrogen action (9), to appraise the feedback actions of endogenous estradiol on spontaneous and exogenous gonadotropin-releasing hormone ( $\mathrm{GnRH}$ )-stimulated pulsatile bioactive LH release. We used the rat interstitial cell testosterone (RICT) bioassay to estimate the quantity of biologically active LH in plasma under these conditions of experimentally perturbed estrogen action.

\section{Methods}

\section{Patient characteristics}

21 normal men (ages 21-29) participated in this study after provision of written informed consent, which was approved by the Human Investigation Committee of the University of Virginia School of Medicine. Each individual had normal serum concentrations of immunoreactive cortisol, thyroxine, prolactin, LH, follicle-stimulating hormone, thyroid-stimulating hormone, equilibrium-dialyzable plasma free testosterone (10), and estradiol (10), and had normal hepatic, renal, and hematological function.

\section{Clinical protocols}

Estradiol infusion. Chromatographically pure estradiol (assessed by high-pressure liquid chromatography) was dissolved in sterile ethanol, which was diluted in $5 \%$ dextrose in water immediately before infusions. 1 liter of $5 \%$ dextrose in water containing $48 \mu \mathrm{g} 17 \beta$-estradiol was infused continuously every $12 \mathrm{~h}$ for $3.5 \mathrm{~d}(10)$. A uniform rate of infusion was maintained with an infusion pump (Volumetric 927; Imed Inc., San Diego, CA), with tygon tubing to minimize nonspecific steroid adsorption, as reported previously (10). All blood sampling was performed in the arm contralateral to the infusion. $1 \mathrm{~d}$ before, and on the $3 \mathrm{rd} \mathrm{d}$ of steady state infusion, blood sampling was initiated at 8 a.m. by withdrawing blood every $4 \mathrm{~min}$ for $8 \mathrm{~h}$ to obtain an integrated 
estimate of bioactive $\mathrm{LH}$, immunoactive $\mathrm{LH}$, and the ratio of biologically active LH to immunoactive LH. This study was performed in seven men.

Administration of the antiestrogen tamoxifen. A group of eight other men received $20 \mathrm{mg}$ tamoxifen orally twice a day for $10 \mathrm{~d}$. To estimate integrated plasma LH concentrations, each individual underwent blood sampling at 20-min intervals for $6 \mathrm{~h}$ under basal conditions (before tamoxifen administration) and after 1, 3, 6, and $10 \mathrm{~d}$ of tamoxifen administration.

Six additional men were studied to evaluate the influence of an antiestrogen on pulsatile $\mathrm{LH}$ release. Based upon the results of the time-course experiments, these individuals received $10 \mathrm{mg}$ tamoxifen every $12 \mathrm{~h}$ for $7 \mathrm{~d}$. Blood sampling was undertaken under basal conditions (before tamoxifen administration) and then again on the 7th $d$ of drug ingestion. Sampling was initiated at 8 a.m. by withdrawing blood at 10-min intervals for $16 \mathrm{~h}$. Tamoxifen was continued during this time, with doses $1 \mathrm{~h}$ before the onset of blood sampling and $12 \mathrm{~h}$ later. The $1 \mathrm{st} 12 \mathrm{~h}$ blood sampling was used to assess spontaneous $\mathrm{LH}$ pulsatility. After this baseline interval of $12 \mathrm{~h}, \mathrm{GnRH}(10 \mu \mathrm{g})$ was injected intravenously. A second dose of $\mathrm{GnRH}(10 \mu \mathrm{g})$ was injected 2 $h$ later. This paradigm was used to test pituitary responsiveness to exogenous pulses of GnRH.

\section{Hormone assays}

Plasma samples were assayed for immunoactive LH by double-antibody radioimmunoassay (RIA), with a sensitivity of $1 \mathrm{mIU} / \mathrm{ml}$ in terms of the Second International Reference Preparation of Human Menopausal Gonadotropin, and for bioactive LH by the RICT bioassay, with a sensitivity of $0.4 \mathrm{mIU} / \mathrm{ml}(1,11)$. The coefficients of variation were $8.4 \%$ (at $2 \mathrm{mIU} / \mathrm{ml}$ ), $6.8 \%$ (at $11.5 \mathrm{mIU} / \mathrm{ml}$ ), and $4.6 \%$ (at 47 $\mathrm{mIU} / \mathrm{ml}$ ) for the RIA, which exhibits $<15 \%$ crossreactivity with alpha subunit. The intraassay coefficient of variation for the bioassay averaged $8.5 \%$ with a range of $8.2-9.2 \%$ in children and postmenopausal women.

\section{Pulse analysis}

To minimize assumptions regarding the shape, frequency, amplitude, or baseline stability of the bioactive LH time-concentration profiles under control or treated conditions, we applied a simple statistical algorithm previously validated for the detection of significant increases or decreases in data series of known variance (12). This method, termed "Cluster" analysis, evaluates successive clusters of designated sizes in relation to an indicated $t$ statistic, marks all significant increases and decreases in the data series, and then designates a peak as a region comprising an increase followed by a decrease. The choice of $t$ statistics permits one to designate an estimated maximal false-positive rate for peak detection assuming Gaussian-distributed measurement error (12). Regions in the data that contain no significant increases or decreases are identified as interpulse "valleys." In the present analyses, the $t$ statistic was selected to maintain a maximal false-positive rate of $1 \%$ by using test cluster sizes of two in the prepeak nadir, peak, and postpeak nadir.

The results of Cluster analysis permit one to quantitate the following properties of pulsatile hormone release: $(a)$ number of statistically significant peaks (pulse frequency); $(b)$ the mean interval between peaks (interpulse interval in min); (c) the mean peak width (duration in min); $(d)$ the maximal peak height $(\mathrm{mIU} / \mathrm{ml}) ;(e)$ the maximal peak height expressed as a percent increase above nadir (fractional amplitude); $(f)$ the peak height expressed as an increment $(\mathrm{mIU} / \mathrm{ml})$ above preceding nadir; $(g)$ the number of valleys (regions without any significant increase or decrease in the data); $(h)$ average valley duration ( $\mathrm{min})$; and ( $i$ ) average valley mean $\mathrm{LH}$ concentration $(\mathrm{mIU} / \mathrm{ml})(12)$. We used the method of Santen and Bardin (13) to determine integrated LH concentrations over the 12 -h baseline sampling.

\section{Statistical analysis}

Within-subject differences were sought by paired two-tailed Student's $t$ testing when mean plasma hormone concentrations were compared.
Because of departures from normality, individual pulse parameters were compared using the Wilcoxon ranked sign test (14). Plasma bio/ immuno LH ratios were compared by parametric methods after logarithmic transformation. Results are expressed as means \pm SEM or median values. Statistical significance was construed for $P \leq 0.05$.

\section{Results}

\section{Steady state infusion of estradiol}

As shown in Fig. $1 A$, during steady state infusion of estradiol mean plasma concentrations of immunoactive and bioactive LH declined significantly $(P=0.013$ and $P=0.016$, respectively). This infusion regimen resulted in an increase in mean serum estradiol concentrations from $32 \pm 4 \mathrm{pg} / \mathrm{ml}$ basally to $64 \pm 5 \mathrm{pg} / \mathrm{ml}$ over the last $8 \mathrm{~h}$ of the infusion $(P<0.01)$. Since we have previously shown that equilibrium is achieved within $24 \mathrm{~h} \mathrm{(10),} \mathrm{this} \mathrm{paradigm} \mathrm{permitted} \mathrm{us} \mathrm{to} \mathrm{appraise} \mathrm{the} \mathrm{influence}$ of steady state plasma estradiol elevations on the bio/immuno LH ratio. In response to estradiol infusion, there was a more pronounced decline in circulating bioactive than immunoactive LH concentrations, which was reflected in a significant decline in plasma bio/immuno $\mathrm{LH}$ ratios in these seven men $(P=0.0097$ Fig. $1 B)$. These measurements of $\mathrm{LH}$ bioactivity and immunoactivity were derived from a pool of aliquots of plasma removed from samples collected at 4-min intervals over $8 \mathrm{~h}$, and as such provide an accurate estimate of integrated plasma LH concentrations over this interval.

\section{Time-dependent influence of antiestrogen administration on plasma bioactive $L H$ concentrations}

Eight men received tamoxifen for $10 \mathrm{~d}$. The 6-h integrated plasma $\mathrm{LH}$ concentrations as assessed by bioassay and immu-
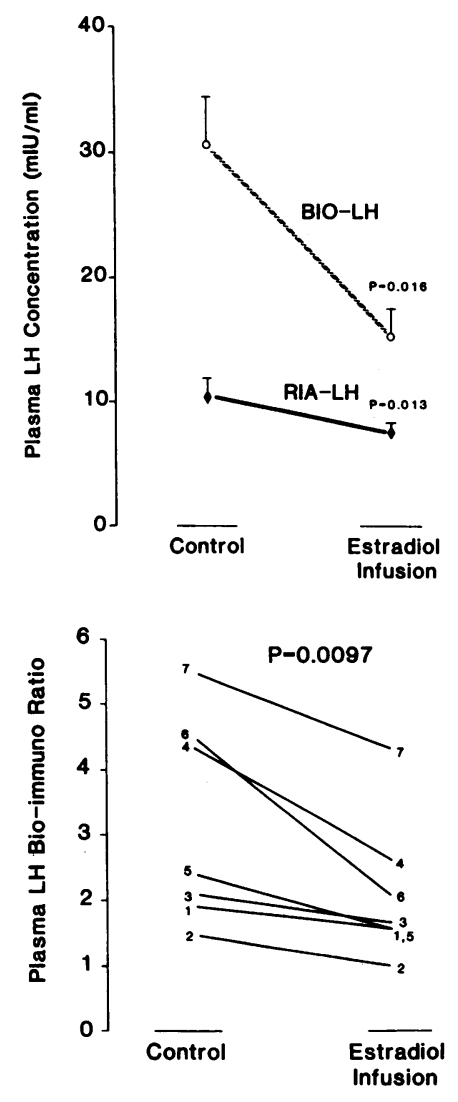

Figure 1. Suppressive effects of steady state estradiol infusion on mean plasma LH concentrations in normal men. Seven normal men received $48 \mu \mathrm{g} / \mathrm{d}$ $17 \beta$-estradiol by continuous intravenous infusion for $3.5 \mathrm{~d}$. (A) Mean plasma concentrations of $\mathrm{LH}$ were determined by bioassay (bio-LH) and immunoassay (RIA-LH) under baseline (control) conditions and during the last $8 h$ of the estradiol infusion. Data represent mean \pm SEM plasma $\mathrm{LH}$ concentrations obtained from pooled aliquots of plasma withdrawn at 4-min intervals for $8 \mathrm{~h}$. (B) Corresponding changes in the plasma bio/immuno $\mathrm{LH}$ ratio in response to steady state estradiol infusion. The numerals in the control and estradiol infusion columns denote individual men. 

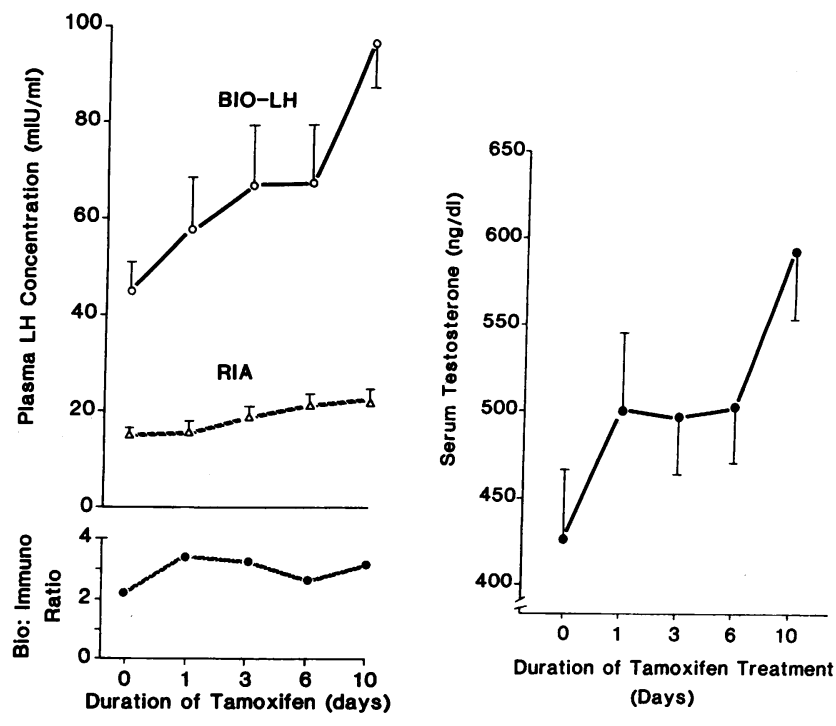

Figure 2. Time course of the stimulatory effects of the antiestrogen, tamoxifen, on mean plasma LH concentrations. Eight normal men underwent repetitive venous sampling (Methods) to estimate mean plasma LH and steroid concentrations basally (Day 0 ) and after 1, 3, 6 , and $10 \mathrm{~d}$ of oral tamoxifen administration. $(A)$ The plasma concentrations of bioactive LH (bio-LH) and immunoactive LH (RIA) are shown as means \pm SEM. The plasma bio/immuno LH ratio is given as the median value. $(B)$ Time-dependent influence of tamoxifen on serum total testosterone in the same men.

noassay are shown in Fig. $2 \mathrm{~A}$. Basal bioactive LH concentrations increased from a mean ( \pm SEM) of $42.7 \pm 6.9 \mathrm{mIU} / \mathrm{ml}$ to a maximal value of $97.6 \pm 19.4 \mathrm{mIU} / \mathrm{ml}(P=0.016)$ after $10 \mathrm{~d}$ of antiestrogen administration. The mean plasma immunoactive LH concentration also increased, although the fractional increase above baseline was of lesser magnitude; namely, basal $15.0 \pm 0.78 \mathrm{mIU} / \mathrm{ml}$ to $21.4 \pm 1.4 \mathrm{mIU} / \mathrm{ml}$ after $10 \mathrm{~d}$ of tamoxifen. Accordingly, the median bio/immuno LH ratios in these men increased significantly, i.e., from 2.1 basally to 3.1 and 3.0 after, respectively, 3 and $10 \mathrm{~d}$ of antiestrogen $(P=0.019)$. Under these conditions, total serum testosterone concentrations also increased significantly (Fig. $2 B$ ).

Impact of antagonism of endogenous estrogen action on 12-h mean concentrations and the pulsatile release of biologically active $\mathrm{LH}$

Mean and integrated plasma LH concentrations. Based upon the preceding dose- and time-course studies, we studied the effects of endogenous estradiol on the pulsatile release of bioactive $\mathrm{LH}$ by sampling blood at 10-min intervals for $16 \mathrm{~h}$ under basal conditions, and again after the administration of tamoxifen for $7 \mathrm{~d}$. During the last $4 \mathrm{~h}$ of sampling, two pulses of GnRH (10 $\mu \mathrm{g})$ were infused intravenously at 2-h intervals to test pituitary responsiveness. The $1 \mathrm{st} 12 \mathrm{~h}$ of sampling was used to evaluate the impact of antiestrogen treatment on spontaneous (endogenous GnRH driven) release of bioactive LH. As shown in Fig. $3 \mathrm{~A}$, the 12-h mean and integrated plasma concentrations of bioactive $\mathrm{LH}$ increased significantly $(P$ $=0.033$ and $P=0.034$, respectively) in response to tamoxifen administration. In contrast, this lower dose of tamoxifen (10 mg every $12 \mathrm{~h}$ ) elicited no significant increase in mean or integrated serum concentrations of immunoactive LH ( $P$ $=0.68$ and $P=0.66$, respectively). Moreover, under these conditions, total serum testosterone concentrations rose significantly $(P=0.004$; Fig. $3 B)$, but plasma free testosterone con-

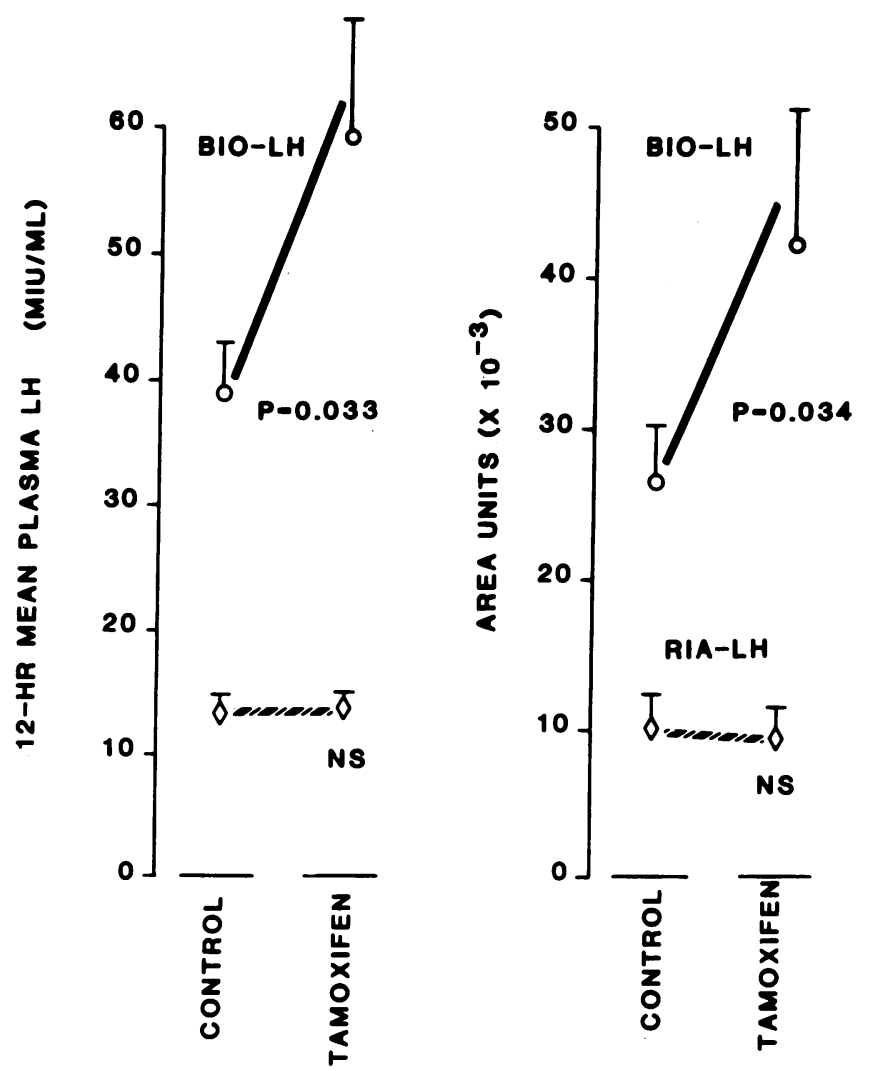

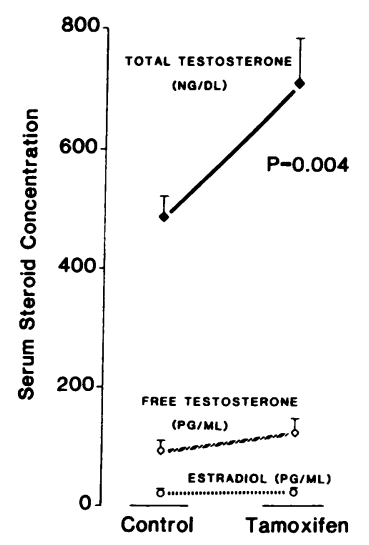

Figure 3. Augmentation of the 12-h mean plasma bioactive LH concentrations in response to administration of the antiestrogen, tamoxifen. Six normal men were studied by repetitive venous sampling (blood withdrawal at 10-min intervals for $12 \mathrm{~h}$ ) under basal conditions (control) and after oral tamoxifen administration (tamoxifen). $(A)$ The subsequent plasma samples were assayed individually to estimate accurately the 12-h mean (left) and integrated plasma $\mathrm{LH}$ concentrations (right). Plasma was analyzed both by bioassay (bio-LH) and immunoassay (RIA-LH). (B) Influence of tamoxifen treatment on serum total testosterone, free testosterone, and estradiol in the same men. Only serum total testosterone increased significantly in response to tamoxifen administration. 
centrations rose only minimally (namely, from $96.4 \pm 11 \mathrm{pg} / \mathrm{ml}$ to $128 \pm 22 \mathrm{pg} / \mathrm{ml}, P=0.19)$. This pattern reflected a slight decrease in the percent dialyzable (free) testosterone in plasma after tamoxifen treatment (percent dialyzable testosterone declined from $1.94 \pm 0.13$ to $1.83 \pm 0.14 ; P=0.23$ ). Mean plasma estradiol concentrations were unchanged, i.e., basal levels were $23.7 \pm 3.0 \mathrm{pg} / \mathrm{ml}$ as compared with $24.2 \pm 3.7 \mathrm{pg} / \mathrm{ml}$ after tamoxifen treatment $(P=0.85)$. Accordingly, the present schedule of antiestrogen administration permitted us to examine changes in plasma bioactive LH release in the absence of any major perturbations in serum concentrations of immunoactive $\mathbf{L H}$, free testosterone, or estradiol.

Effects of antiestrogen on specific properties of pulsatile bioactive $\mathrm{LH}$ release. The plasma collected at 10 -min intervals for $12 \mathrm{~h}$ under basal and tamoxifen-treated conditions allowed us to assess the effect of antiestrogen on specific properties of pulsatile bioactive $\mathrm{LH}$ release. Using the validated Cluster analysis method adjusted to an estimated maximal false-positive rate of $1 \%$, we observed that bioactive $\mathrm{LH}$ pulse frequency (number of pulses counted per $12 \mathrm{~h}$ ) increased significantly in conjunction with antiestrogen administration; namely, from $5.3 \pm 0.33$ pulses/ $12 \mathrm{~h}$ (median 5.5 ) basally to $7.5 \pm 0.22$ pulses/ $12 \mathrm{~h}$ (median 7.5) after tamoxifen $(P=0.029$; Fig. $4 A){ }^{2}$ Correspondingly, the basal bioactive $\mathrm{LH}$ interpulse interval of $113 \pm 7.9$ min (median 105 ) declined to $85 \pm 2.6$ min (median $84)$ in association with antiestrogen treatment $(P=0.029$ by nonparametric testing; Fig. $4 \mathrm{~B}$ ).

Administration of a dose of antiestrogen sufficient to increase mean and integrated plasma concentrations of bioactive LH also elicited significant and distinct changes in LH pulse properties. As shown in Fig. 5, the duration of bioactive $\mathrm{LH}$ peaks decreased significantly from a median value of 99 to 71 min during tamoxifen administration $(P=0.029)$. This occurred in association with a significant increase in absolute (maximal) amplitude of the bioactive LH peak, which rose from a median value of 50 to $65 \mathrm{mIU} / \mathrm{ml}(P=0.029)$. Thus, tamoxifen had reciprocal effects on peak width (which decreased) and a maximal peak height (which increased). This interaction resulted in no significant changes in mean bioactive LH peak area (milliinternational units per milliliter times minute), which was $1,000 \pm 130$ (basal) and $900 \pm 180$ (after tamoxifen) $(P=0.46)$.

Mean interpulse valley (region of nonpulsatility) bioactive LH concentrations also increased significantly during tamoxifen administration, namely, from $32 \pm 5.4$ to $49 \pm 11 \mathrm{mIU} / \mathrm{ml}$ $(P=0.02$ over basal); whereas, incremental LH pulse amplitudes did not change significantly (Fig. 5; median basal 18 $\mathrm{mIU} / \mathrm{ml}$ and posttamoxifen $23 \mathrm{mIU} / \mathrm{ml}$ ). Also, the duration (minutes) of the interpulse valleys was not altered significantly by antiestrogen: interpulse valley durations of $30 \pm 4 \mathrm{~min}$ (basal) vs. $31 \pm 0.68 \mathrm{~min}$ (posttamoxifen) $(P=0.079)$.

\section{Profiles of bio- and immunoactive $\mathrm{LH}$ release in control and antiestrogen treatment sessions}

Individual profiles of bio- and immunoactive $\mathrm{LH}$ release are shown in Fig. 6, in which control and tamoxifen treatment

2. LH pulse frequency per se may overestimate the interpulse interval because of regions of nonpulsatility at the begining and end of the sampling interval. This "edge" artifact does not occur for measured interpulse intervals.
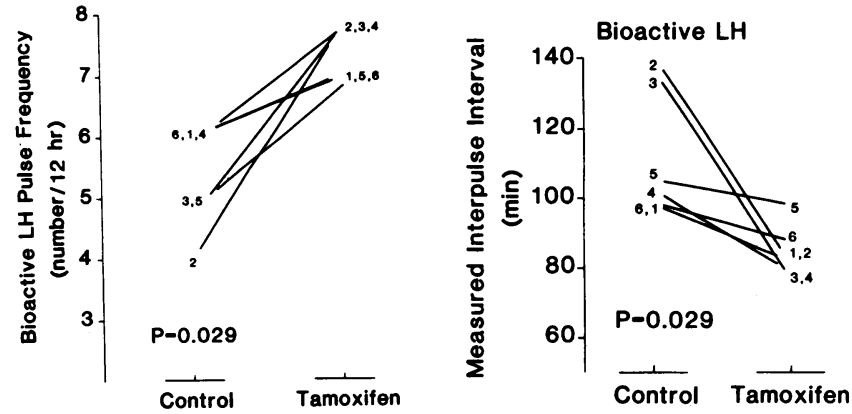

Figure 4. Influence of the antiestrogen, tamoxifen, on bioactive $\mathrm{LH}$ pulse frequency (pulses/12 h) $(A)$ and interpulse interval (minute) $(B)$. Six normal men were studied by repetitive venous sampling over $12 \mathrm{~h}$. Computerized analysis was applied to the bioactive LH concentration-time series to enumerate statistically significant pulses.

sessions are given for two men. Note the preferential increase in bioactive $\mathrm{LH}$ in response to low-dose exogenous GnRH pulses (discussed further below).

\section{Influence of antiestrogen treatment on exogenous $\mathrm{GnRH}$ - stimulated release of bioactive $\mathrm{LH}$}

To test the impact of antiestrogen administration on pituitary responsiveness to exogenous GnRH, two consecutive pulses of synthetic GnRH ( $10 \mu \mathrm{g}$ each) were administered intravenously at 2-h intervals. The hourly mean plasma concentrations of bio- and immunoactive $\mathrm{LH}$ were compared in basal and tamoxifen-treated sessions. As shown in Fig. 7 (top), mean baseline (pre-GnRH) plasma bioactive LH concentrations were increased significantly in the tamoxifen-treated group ( $P$ $=0.023$ and $P=0.001$, respectively, for the first and second baseline hours). In response to the first and second injections of $\mathrm{GnRH}$, mean plasma bioactive LH concentrations increased significantly in both the control and tamoxifen-treated sessions $(P<0.001)$. However, the degree of increase in plasma bioactive $\mathrm{LH}$ concentrations in response to two consecutive exogenous $\mathrm{GnRH}$ pulses was significantly reduced in the tamoxifen treatment session as compared with control $(P$

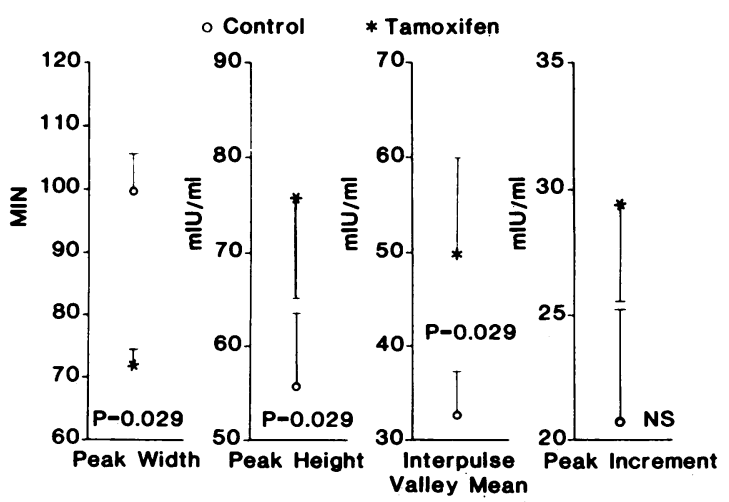

Figure 5. Influence of the antiestrogen, tamoxifen, on distinct properties of bioactive LH pulses. Six normal men underwent repetitive venous sampling over $12 \mathrm{~h}$ for subsequent analysis of bioactive $\mathrm{LH}$ pulsatility. Data are means \pm SEM for control or tamoxifen treatment sessions in relation to bioactive LH peak width (duration), maximal peak height, interpulse valley mean bioactive LH concentration, and bioactive $\mathrm{LH}$ peak increment. 

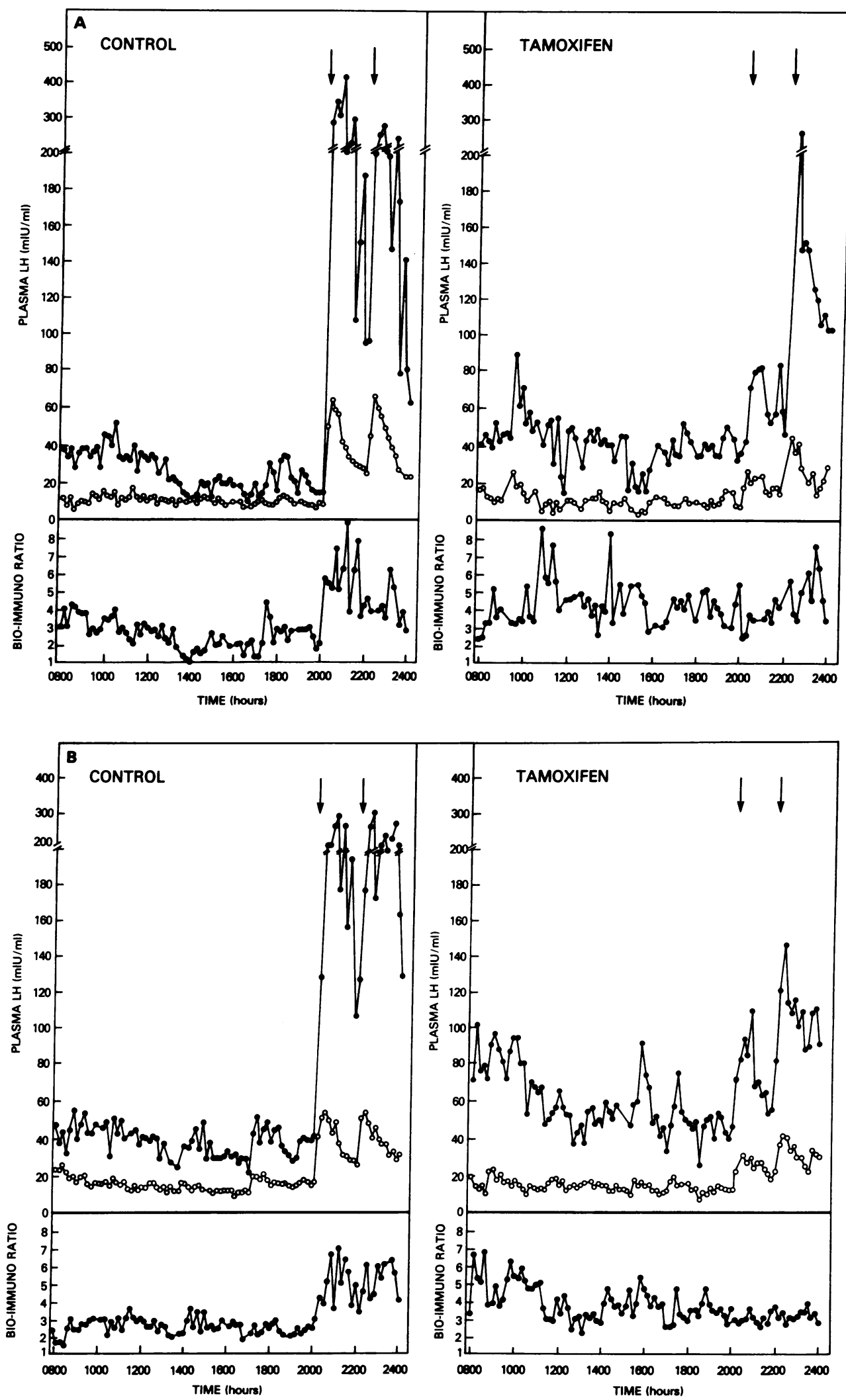

Figure 6. Illustrative profiles of bioand immunoactive $\mathrm{LH}$ release in two normal men in control sessions and after treatment with the antiestrogen, tamoxifen. In each subject ( $A$ and $B$ ), bioactive LH (top, solid circles), immunoactive LH (top, open circles), and bio/immuno LH ratios (bottom) are given for plasma collected at 10-min intervals for 16 $\mathrm{h}$. The $1 \mathrm{st} 12 \mathrm{~h}$ of basal sampling were followed by paired, 2-h intravenous injections of GnRH ( $10 \mu \mathrm{g}$ each) at the times denoted by the vertical arrows. Note break in ordinate scale to accommodate the marked increases in bioactive $\mathrm{LH}$ in response to exogenous GnRH.

$<0.004$ to $P<0.014$ for hours $3-6$ ). A similar pattern of relatively suppressed $\mathrm{LH}$ responses to $\mathrm{GnRH}$ after tamoxifen administration was observed for immunoactive LH (Fig. 7, middle). We noted that for both immuno- and bioactive $\mathrm{LH}$, tamoxifen treatment resulted in greater suppression of the maximal response to the first rather than second pulse of GnRH $(P<0.02)$.

Under basal conditions (no tamoxifen), hourly median plasma bio/immuno LH ratios increased significantly in response to exogenous pulses of GnRH $(P<0.001)$ (Fig. 7, bot- 

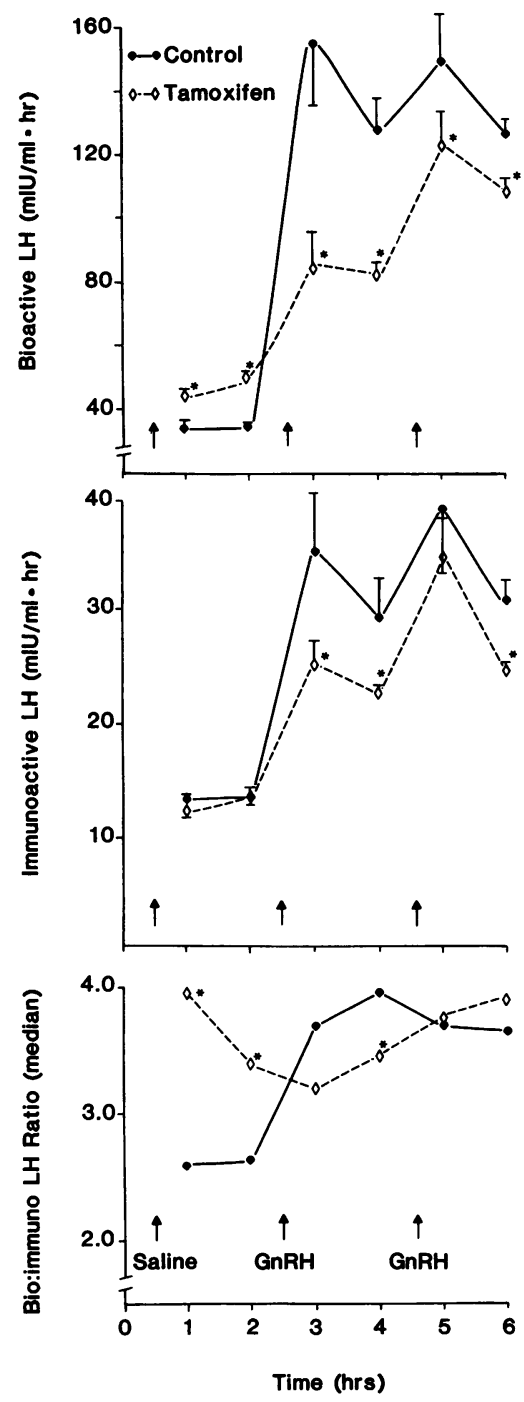

Figure 7. Impact of the antiestrogen, tamoxifen, on exogenous GnRHstimulated bio- and immunoactive $\mathrm{LH}$ release. Six normal men were studied under basal (control) conditions and after the administration of tamoxifen. To test pituitary responsiveness, saline vehicle or two exogenous pulses of GnRH (10 $\mu \mathrm{g})$ were administered intravenously at 2-h intervals, as indicated in the bottom. The time courses for plasma bioactive LH (top), immunoactive LH (middle) and the bio/immuno LH ratio (bottom) are depicted. Data are means \pm SEM derived from samples collected at 10 -min intervals over the preceding $1 \mathrm{~h}(n$ $=6$ ). In the case of the bio/immuno LH ratio, the median values are shown. Asterisks designate significant differences between control and tamoxifen sessions at that hour.

tom). When antiestrogen was administered, baseline (preGnRH) median bio/immuno LH ratios increased significantly above control levels (hours 1 and $2, P=0.003$ ). However, stimulation with two consecutive pulses of $\mathrm{GnRH}$ did not result in any further increase in the plasma bio/immuno LH ratio during tamoxifen treatment. Rather, the median plasma bio/immuno $\mathrm{LH}$ ratios were significantly reduced $2 \mathrm{~h}$ after the second GnRH pulse (hour $4, P=0.036$ ).

\section{Analysis of the impact of antiestrogen administration on} intra- and interpulse plasma bio/immuno $\mathrm{LH}$ ratios in men

In both control and tamoxifen treatment sessions, the plasma bio/immuno $\mathrm{LH}$ ratios were significantly higher within spontaneous pulses than in the corresponding interpulse intervals ( $P=0.01$ in the control session, and $P=0.008$ in the tamoxifen treatment session; Fig. 8). Tamoxifen treatment resulted in a significant increase in both spontaneous inter- and intrapulse bio/immuno LH ratios $(P=0.008$ and $P=0.003$, respectively). In contrast, antiestrogen administration attenuated the ability of exogenous GnRH to increase the bio/immuno LH ratio further, which suggests the attainment of maximal enrichment in endogenous LH bioactivity in the presence of antiestrogen.

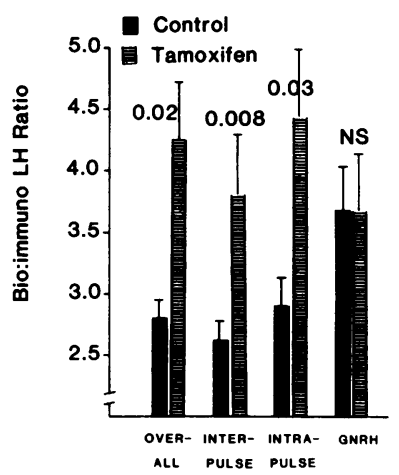

Figure 8. Influence of the antiestrogen, tamoxifen, on plasma bio/ immuno LH ratios. "Overall" plasma bio/immuno $\mathrm{LH}$ ratios designate values observed over 12 h of basal sampling before GnRH injection. "Interpulse" and "intrapulse" values apply to plasma bio/ immuno LH ratios observed during the interpulse (valley) intervals and within actual bioactive $\mathrm{LH}$ pulses, respectively. The "GnRH" bio/immuno $\mathrm{LH}$ ratios were determined during the $4 \mathrm{~h}$ when

two pulses of exogenous GnRH were administered. Data are means \pm SEM for the corresponding individual median bio/immuno LH ratios in six men. The decimal values above the bar graphs denote the degree of significant difference between control and tamoxifen treatment sessions (NS, $P>0.05$ ).

\section{Discussion}

The present investigations demonstrate significant and distinct modulatory actions of estradiol on the pulsatile secretion of LH molecules enriched in biological activity. In particular, steady state intravenous infusion of estradiol at a dosage that mimics its endogenous production rate (10) preferentially suppressed mean circulating bioactive LH concentrations, with a consequent significant decline in the plasma bio/immuno $\mathrm{LH}$ ratio. Conversely, administration of a selective antiestrogen disproportionately increased integrated plasma concentrations of bioactive over immunoactive LH. Since these complementary strategies were not associated with any significant alterations in plasma free testosterone concentrations, we infer that observed changes in circulating bioactive LH concentrations can be attributed to corresponding changes in estrogen action per se. As such, the present studies provide the first direct evidence, to our knowledge, that estradiol can modulate circulating concentrations of biologically active $\mathbf{L H}$ in normal men.

After identifying a dose of tamoxifen that resulted in a selective augmentation of plasma bioactive LH concentrations, we were able to examine the physiological mechanisms subserving this increase. Computerized analysis of bioactive LH pulsations revealed that antagonism of endogenous estradiol feedback with the antiestrogen, tamoxifen, elicited a significant increase in $\mathrm{LH}$ pulse frequency with a commensurate decline in the LH interpulse interval. These changes occurred despite a decrease in pituitary responsiveness to exogenous pulses of GnRH. Thus, assuming that there is a temporal relationship between the frequency of hypothalamic $\mathrm{GnRH}$ release and that of endogenous LH pulses $(15,16)$, the present results suggest that tamoxifen can activate neural timing mechanism(s) that govern the intermittent release of endogenous GnRH. This inference is consistent with the ability of other antiestrogens to stimulate GnRH release from hypothalamic tissue in vitro (17) and in some, but not all cases to increase immunoactive LH pulsatility in vivo (18-20). Most importantly, the present use of the RICT bioassay of LH suggests a physiological role for endogenous estradiol in men, such that secreted and/or extragonadally synthesized estrogen constrains secretion of $\mathrm{LH}$ molecules enriched in biological activity. 
The present use of a statistically based pulse detection algorithm also revealed distinct changes in individual bioactive $\mathrm{LH}$ pulse properties when endogenous estrogen action was antagonized. Antiestrogen administration increased not only mean plasma bioactive $\mathrm{LH}$ concentrations and bioactive $\mathrm{LH}$ pulse frequency, but also augmented maximal bioactive LH peak amplitude and interpulse valley mean LH pulse concentrations (bioactive LH concentrations observed in plasma during intervals with no detectable pulsatility). The higher frequency of pulsatile bioactive $\mathrm{LH}$ release was associated with diminished bioactive LH peak duration. Since peak duration decreased but peak amplitude increased, there was no net change in mean bioactive LH peak area. To our knowledge, these significant changes in specific properties of the bioactive $\mathrm{LH}$ pulse signal provide the first evidence that endogenous estrogen feedback influences certain characteristics of the bioactive LH pulse, namely amplitude, pulse duration, and interpulse (valley) hormone concentrations. Although the exact impact of such changes in bioactive LH pulse properties on the gonad are not known, the frequency and amplitude characteristics of endocrine signals can substantially influence target tissue responses. Accordingly, the ability of estradiol to modulate specific properties of the LH pulse signal as well as its frequency may have significant implications in relation to target tissue function.

The ability of estradiol to modulate the secretion of $\mathrm{LH}$ molecules enriched in biological activity may also reflect direct actions of estradiol on gonadotrope function, since estrogen receptors have been identified in anterior pituitary cells and estradiol is capable of directly modulating $\mathrm{LH}$ release from perifused pituitary glands (21). In this regard, estradiol may influence the cellular processing and/or one or more aspects of terminal glycosylation of LH molecules (22).

Earlier regimens using a single large bolus of $\mathrm{GnRH}$ and/or continuous GnRH infusions were not able to disclose a stimulatory effect of exogenous $\mathrm{GnRH}$ on the plasma bio/immuno LH ratio (23-25). These findings might be explained by functional compartmentalization of releasable $\mathrm{LH}$ pools, such that pharmacological GnRH stimulation increases secretion from all pituitary $\mathrm{LH}$ pools yielding an integrated bio/immuno value, rather than selective release from a highly bioactive pool. Also, during continuous GnRH infusion, there may be lack of definition of a small early pool (presumably of high bioactivity) and/or admixture with a late pool of reduced bioactivity. In contrast, the present schedule of two consecutive submaximal pulses of exogenous GnRH did result in an immediate and preferential release of bioactive $\mathrm{LH}$ within a consequent increase in the plasma bio/immuno $\mathrm{LH}$ ratio. This pattern is consistent with the observation that plasma bio/immuno LH ratios in spontaneous (endogenous $\mathrm{GnRH}$ driven) bioactive LH pulses are significantly higher than those in the corresponding interpulse baseline intervals (reference 26 and this report). Furthermore, the present work indicates that antiestrogen treatment can further increase intrapulse bio/immuno LH ratios to a significant degree. Similar facilitation of pulsatile bioactive $\mathrm{LH}$ release could be observed when brain opiate inhibitory tone was opposed by naltrexone (26). Thus, we infer that spontaneous LH pulses, putatively generated in response to endogenous GnRH stimulation of gonadotropes, exhibit a relative enrichment in biological activity, and this degree of enrichment can be modulated by estrogen action. Moreover, we suggest that this physiological mode of episodic bioactive LH release may serve to minimize gonadal desensitization that might occur if similar concentrations of highly bioactive $\mathrm{LH}$ were secreted in a constant manner.

Although endogenous LH production rates have not yet been measured in response to estrogen infusion or antiestrogen administration in man, the present results would be consistent with a suppressive effect of estradiol infusion and a stimulatory effect of antiestrogen administration on endogenous LH production. Moreover, endogenous estrogen excess has been reported to profoundly suppress bioactive LH release (27). Accordingly, the changes we observed in plasma bio/immuno LH ratios might reflect a property of pituitary gonadotropes to secrete LH molecules relatively enriched in bioactivity whenever production rates are increased and to secrete LH molecules relatively impoverished in biological activity whenever LH secretion rates are suppressed. This hypothesis would accord with the striking rise in circulating $\mathrm{LH}$ bioactivity observed in castrate or postmenopausal individuals, in whom LH production rates are increased (7). However, this interpretation would not account for the recent observation that nonsteroidal suppression of endogenous LH secretion with a potent and selective $\mathrm{GnRH}$ antagonist results in a parallel decline in plasma bio- and immunoactive LH concentrations with no discernible variation in the plasma bio/immuno $\mathrm{LH}$ ratio over time (28). Alternatively, estrogen or antiestrogen might affect the metabolic clearance of LH. However, available data suggest that sex steroid hormones do not have a major influence on gonadotropin clearance in man $(29,30)$. Moreover, alterations in metabolic clearance would not account for enhanced bioactive LH pulse frequency and attenuated GnRH-stimulated LH release in the presence of tamoxifen. Accordingly, we infer that estradiol modulates the secretion of bioactive LH by mechanisms that are not dependent upon altered hormone secretion or clearance rates per se.

In summary, we have utilized two complementary strategies to appraise the influence of estradiol on the secretion of biologically active LH in man. Our results with steady state estradiol infusion and a selective antiestrogen indicate that endogenous estradiol modulates the pulsatile secretion of $\mathrm{LH}$ molecules enriched in biological activity. Such observations suggest a critical role for estradiol in regulating the functional attributes of the pituitary-gonadal axis in man.

\section{Acknowledgments}

We thank Chris McNett for her skillful preparation of the manuscript, Paula P. Azimi for the artwork, the National Hormone and Pituitary Program for the provision of purified human LH, and Sandra Jackson and the expert nursing staff at the Clinical Research Center at the University of Virginia for conduct of the research protocols.

This work was supported in part by National Institutes of Health (NIH) Grant RR-00847 to the Clinical Research Center of the University of Virginia, Research Career Development Award 1 K04 HD-00634 (JDV), Diabetes and Research Training Center Grant 5 P60 AM-22125-05, and NIH-supported Clinfo Data Reduction Systems.

\section{References}

1. Dufau, M. L., R. Pock, A. Neubauer, and K. J. Catt. 1976. In vitro bioassay of $\mathrm{LH}$ in human serum: the interstitial cell testosterone (RICT) assay. J. Clin. Endocrinol. \& Metab. 42:958-968.

2. Solano, A. R., M. L. Dufau, and K. J. Catt. 1979. Bioassay and radioimmunoassay of serum luteinizing hormone in the male rat. Endocrinology. 105:372-381. 
3. Marut, E. L., R. F. Williams, B. D. Cowan, A. Lynch, S. P. Lerner, and G. D. Hodgen. 1981. Pulsatile pituitary gonadotropin secretion during maturation of the dominant follicle in monkeys: estrogen positive feedback enhances the biological activity in LH. Endocrinology. 109:2270-2272.

4. Rajalakshmi, M., D. M. Robertson, S. K. Choi, and E. Diczfalusy. 1979. Biologically active luteinizing hormone (LH) in plasma. III. Validation of the in vitro bioassay when applied to male plasma and the possible role of steroidal precursors. Acta Endocrinol. 90:585-598.

5. Solano, A. R., A. Garcia Vela, K. J. Catt, and M. L. Dufau. 1980. Modulation of serum and pituitary luteinizing hormone bioactivity by androgen in the rat. Endocrinology. 106:1941-1948.

6. Dufau, M. L., J. D. Veldhuis, F. Fraioli, M. L. Johnson, and I. Z. Beitins. 1983. Mode of bioactive luteinizing hormone secretion in man. J. Clin. Endocrinol. \& Metab. 57:993-1000.

7. Lucky, A. W., R. W. Rebar, R. L. Rosenfield, N. Roche-Bender, and J. Helke. 1979. Reduction of the potency of luteinizing hormone by estrogen. New Engl. J. Med. 300:1034-1036.

8. Veldhuis, J. D., I. Z. Beitins, M. L. Johnson, M. A. Serabian, and M.L. Dufau. 1984. Biologically active luteinizing hormone is secreted in episodic pulsations that vary in relation to stage of the menstrual cycle. J. Clin. Endocrinol. \& Metab. 58:1050-1058.

9. Sutherland, R., Y. Meister, and E. E. Baulieu. 1977. Tamoxifen is a potent pure antiestrogen in chick oviduct. Nature (Lond.). 267:434.

10. Veldhuis, J. D., A. D. Rogol, E. Samojlik, and N. H. Ertel. 1984. Role of endogenous opiates in the expression of negative feedback actions of androgen and estrogen on pulsatile properties of luteinizing hormone secretion in man. J. Clin. Invest. 74:47-55.

11. Dufau, M. L., I. Beitins, J. McArthur, and K. J. Catt. 1977. Bioassay of serum LH concentration in normal and LHRH stimulated human subjects. In The Testis in Normal and Infertile Men. P. Troen and H. R. Nakin, editors. Raven Press, New York. 309-326.

12. Veldhuis, J. D., and M. L. Johnson. 1986. Cluster analysis: a simple, versatile and robust algorithm for endocrine pulse detection. Am. J. Physiol. 250:E486-E493.

13. Santen, R. J., and C. W. Bardin. 1973. Episodic luteinizing hormone secretion in man. Pulse analysis, clinical interpretation, physiologic mechanisms. J. Clin. Invest. 52:2617-2628.

14. Winer, B. J. 1971. Statistical Principles in Experimental Design. McGraw-Hill Publications, New York. 300 pp.

15. Clarke, I. J., and J. T. Cummins. 1982. The temporal relationship between gonadotropin releasing hormone (GnRH) and luteinizing hormone (LH) secretion in ovariectomized ewes. Endocrinology. 111:1737-1740.

16. Levine, J. E., and V. D. Ramirez. 1982. Luteinizing hormonereleasing hormone release during the rat estrous cycle and after ovariectomy, as estimated with push-pull cannulae. Endocrinology. 111:1439-1444.

17. Miyake, A., K. Tasaka, T. Sakumoto, Y. Kawamura, Y. Nagahara, and T. Aono. 1983. Clomiphene citrate induces luteinizing hor- mone release through hypothalamic luteinizing hormone-releasing hormone in vitro. Acta Endocrinol. 103:289-292.

18. Winters, S. J., and P. Troen. 1985. Evidence for a role of endogenous estrogen in the hypothalamic control of gonadotropin secretion in men. J. Clin. Endocrinol. \& Metab. 61:842-845.

19. Boyar, R. M., M. Perlow, S. Kapen, G. Lefkowitz, E. Weitzman, and L. Hellman. 1973. The effect of clomiphene citrate on the 24-hour LH secretory pattern in normal men. J. Clin. Endocrinol. \& Metab. 36:561-566.

20. Santen, R. J., and E. R. Ruby. 1979. Enhanced frequency and magnitude of episodic luteinizing hormone-releasing hormone discharge as a hypothalamic mechanism for increased luteinizing hormone secretion. J. Clin. Endocrinol. \& Metab. 48:315-319.

21 . Drouin, J., and F. Labrie. 1981. Interactions between $17 \beta$-estradiol and progesterone in the control of luteinizing and follicle stimulating hormone release in rat anterior pituitary cells in culture. Endocrinology. 108:52-57.

22. Pollack, L., and P. Atkinson. 1983. Correlation of glycosylation forms with position in amino acid sequence. J. Cell Biol. 97:293-312.

23. Dufau, M. L., I. Z. Beitins, J. W. McArthur, and K. J. Catt. 1976. Effects of luteinizing hormone-releasing hormone (LHRH) upon bioactive and immunoreactive serum LH levels in normal subjects. J. Clin. Endocrinol. \& Metab. 43:658-667.

24. Beitins, I. Z., M. L. Dufau, K. O'Loughlin, K. J. Catt, and J. W. McArthur. 1977. Analysis of biological and immunological activities in the two pools of $\mathrm{LH}$ released during constant infusion of luteinizing hormone-releasing hormone (LHRH) in men. J. Clin. Endocrinol. \& Metab. 45:605-608.

25. Lobo, R. A., D. Shoupe, S. P. Chang, and J. Campeau. 1984. The control of bioactive luteinizing hormone secretion in women with polycystic ovary syndrome. Am. J. Obstet. Gynecol. 148:423-428.

26. Veldhuis, J. D., A. D. Rogol, M. L. Johnson, and M. L. Dufau. 1983. Endogenous opiates modulate the pulsatile secretion of biologically active luteinizing hormone in man. J. Clin. Invest. 72:20312040.

27. Veldhuis, J. D., J. R. Sowers, A. D. Rogol, and M. L. Dufau. 1985. Pathophysiology of male hypogonadism associated with endogenous hyperestrogenism: evidence for dual defects in the gonadal axis. New Engl. J. Med. 312:1371-1375.

28. Davis, M., J. D. Veldhuis, A. D. Rogol, M. L. Dufau, and K. J. Catt. 1987. Sustained inhibitory actions of a novel antagonist of gonadotropin-releasing hormone $(\mathrm{GnRH})$ in man. J. Clin. Endocrinol. \& Metab. 64:1268-1274.

29. Wehmann, R. E., M. R. Blackman, and S. M. Harman. 1982. Metabolic clearance rates of luteinizing hormone in women during different phases of the menstrual cycle and while taking an oral contraceptive. J. Clin. Endocrinol. \& Metab. 55:654-659.

30. Kohler, P. O., G. T. Ross, and W. D. Odell. 1968. Metabolic clearance and production rates of human luteinizing hormone in preand postmenopausal women. J. Clin. Invest. 47:38-47. 